\title{
AVALIAÇÃO DA QUALIDADE NUTRICIONAL DAS REFEIÇÕES SERVIDAS AOS IDOSOS EM INSTITUIÇÃO ASILAR ${ }^{1}$
}

\author{
Ediana Nogueira de Carvalho ${ }^{2}$ \\ Francisca Rosângela da Silva ${ }^{3}$ \\ Martha Teresa Siqueira Marques Melo ${ }^{4}$ \\ Cecilia Maria Resende Gonçalves de Carvalho
}

\section{Resumo}

Estudou-se a alimentação dos idosos residentes em três instituições geriátricas de Teresina-PI para avaliar a qualidade nutricional das refeições servidas. Na determinação da porção média dos alimentos e preparações foi utilizado o método da pesagem direta. Os valores calórico e nutritivo dos alimentos ou preparações servidas aos idosos foram determinados através do programa computadorizado de cálculo de dietas desenvolvido pela Universidade Federal de São Paulo. A adequação de calorias, proteínas, ferro e vitamina $\mathrm{C}$ foi feita com base nos parâmetros do National Council Research preconizadas nas Recommended Dietary Allowances (1989), enquanto a adequação de cálcio e fósforo foi executada de acordo com Recommended Dietary Intake (1998). Os resultados indicaram consumo calórico inadequado em duas instituições, apresentando excesso em uma e deficiência em outra (146,42\% e 75,68\%), e ingestão protéica acima do recomendado em todas $(112,23 \%, 218,88 \%$ e $122,32 \%)$. O consumo de cálcio foi baixo $(63,57 \%)$ em uma instituição e adequado nas demais, enquanto o de vitamina $\mathrm{C}$ apresentou-se baixo em todas. $\mathrm{O}$ consumo de ferro e de fósforo foi

\footnotetext{
${ }^{1}$ Trabalho elaborado com apoio do Programa de Iniciação Científica da Universidade Federal do Piauí.

${ }^{2}$ Bolsista do Curso de Nutrição da Universidade Federal do Piauí.

${ }^{3}$ Bolsista do Curso de Nutrição da Universidade Federal do Piauí.

${ }^{4}$ Professora do Departamento de Nutrição - Centro de Ciências da Saúde - Universidade Federal do Piauí. Especialização em Nutrição Social.

${ }^{5}$ Professora Adjunta do Departamento de Nutrição - Centro de Ciências da Saúde - Universidade Federal do Piauí - Campus Universitário Petrônio Portela - Teresina/PI. Coordenadora do Núcleo de Pesquisa e Extensão Universitária para a Terceira Idade - Doutora em Ciência da Nutrição. Especialista em Gerontologia Social. E-mail: cecilia@webone.com.br
} 
elevado, apresentando valores bem acima dos recomendados. A inadequação das dietas oferecidas sugere a necessidade de medidas de intervenção a curto prazo, visto que os desequilíbrios alimentares trazem conseqüências nefastas ao estado de saúde, principalmente dos idosos.

Palavras-chave: Idoso. Dieta. Adequação Nutricional. Instituição Asilar.

\section{Introdução}

É surpreendente o fenômeno de crescimento do número de idosos, já superando, atualmente, aquele da população total. As estimativas para o ano 2020 apontam para um contingente de 1,2 bilhões de pessoas acima de 60 anos em todo o mundo, com três quartos desta população vivendo nos países em desenvolvimento. No Brasil, as projeções demográficas do Instituto Brasileiro de Geografia e Estatística indicam um aumento significativo na taxa de crescimento do segmento longevo, com um índice de 5,87\% a cada dez anos (2000). Até 2025 está prevista uma participação de idosos na população brasileira de $15 \%$, com um número estimado de 32 milhões de pessoas com 60 anos ou mais (BRASIL, 1996).

No Piauí, nas últimas décadas, o envelhecimento da população na faixa etária de 60 anos ou mais se expandiu, passando de $6,6 \%$ para 8,3\% entre os anos de 1991 e 2000 (IBGE, 2000).

$\mathrm{O}$ alcance da longevidade representa uma grande conquista para a humanidade, mas com o avançar da idade muitas modificações orgânicas acontecem, afetando o metabolismo, a ingestão de alimentos, a atividade física e o risco de desenvolver doenças (ORTEGA et al., 1992). As desordens de natureza nutricional são muito freqüientes na população idosa e as doenças crônicas não-transmissíveis, também prevalentes nesta faixa etária, fazem com que esses indivíduos necessitem cada vez mais de constantes cuidados e atenção, em virtude do risco de morbidade e de mortalidade que apresentam.

Cerca de $40 \%$ das pessoas com mais de 65 anos de idade estão nas instituições de longa permanência (GUIZOG, 1996) e a cada dia aumenta esse número, o que acarreta aumento do risco de perda de qualidade no padrão de atendimento, fato este negativo, visto que se trata de uma população fragilizada que requer cuidado especializado (BORN, 1997).

A situação de muitos idosos institucionalizados é preocupante, visto que muitos cuidadores e outros profissionais demonstram pouca ou nenhuma ha- 
bilidade com a alimentação deles. Obesidade, desnutrição e desequilíbrios alimentares são sempre mais elevados em idosos institucionalizados, quando comparados aos idosos que ficam fora de instituições. O nível de desnutrição entre eles é significativamente mais elevado em cerca de 30-40\% (GUIZOG, 1996).

Muitos são os obstáculos para o alcance de boa nutrição para o idoso institucionalizado. Idade avançada, condições de saúde frágil quando ingressam nas instituições, abandono pela família, situação financeira ruim das instituições, atitudes negativas no trato com o idoso, ausência do nutricionista no local e leis contratuais da instituição podem ser causas para o alto nível de desnutrição entre eles. Pesquisas realizadas por Marucci (1985) e por Gloth et al. (1996) mostram ingestão insuficiente de muitos nutrientes por idosos institucionalizados.

Atitudes negativas podem contribuir significativamente para a negligência alimentar e comprometer a saúde do idoso. Ainda é predominante na sociedade ocidental a concepção de que envelhecer significa apenas apresentar perdas, prejuízos, decadência e incapacidades. Essas concepções levam à desvalorização da experiência de vida dos mais velhos e, quase sempre, culminam em trajetórias para institucionalizar o idoso em ambientes sombrios, decadentes, sem estímulos, distantes da realidade exterior, permitindo apenas a sua sobrevivência.

O idoso institucionalizado apresenta peculiaridades importantes e, por isso, as instituições devem estar preparadas em termos de recursos humanos, quer seja em quantidade, habilidade ou preparo ideal.

No Piauí, as dificuldades são grandes, seja pela falta de mão-de-obra com o mínimo de qualificação, seja pelas dificuldades financeiras, associadas ao estado de debilidade das condições de saúde e de nutrição e pela situação de abandono de muitos idosos que chegam às instituições.

A alimentação do idoso ainda é muito pouco estudada e a promoção de uma alimentação saudável deve estar inserida na rotina das instituições. $\mathrm{O}$ objetivo deste trabalho é discutir a problemática, avaliando as refeições servidas aos idosos institucionalizados e apresentando propostas para introduzir práticas alimentares corretas e manter o idoso em cuidado nutricional rotineiro e permanente.

A nutrição, em combinação com outras medidas, aumenta a expectativa de vida com qualidade, pois pode atuar como fator de prevenção e controle de várias doenças. Por outro lado, quando não conduzida de forma 
equilibrada e adequada, pode contribuir para o aparecimento e a evolução de enfermidades degenerativas e distúrbios associados ao envelhecimento.

\section{Metodologia}

No município de Teresina, as instituições asilares oficiais são em número de três, atendendo cerca de 156 pessoas na faixa etária de 60 a 95 anos, de um total de mais de 40.000 habitantes com idade maior ou igual a 60 anos. A Instituição 1 atende 46 idosos, com média de idade de $80 \pm 3,3$ anos, e recebe apoio do governo estadual por meio do Serviço Social do Estado, além de doações e trabalhos voluntários. A Instituição Pública 2 recebe apoio do Serviço Social do Estado e atende 79 idosos, com média de idade de $76 \pm 2,7$ anos. A Instituição Asilar 3, de caráter filantrópico, acomoda 31 idosos, com média de idade de $77 \pm 3,8$ anos, e reúne voluntários e profissionais para, em conjunto, promover o atendimento ao idoso. Tem como base de seus recursos a contribuição das aposentadorias dos idosos e o serviço médico do Estado.

\subsection{Avaliação Dietética}

Para avaliar o consumo alimentar dos idosos foi solicitado o consentimento da direção das instituições. Antes da distribuição das refeições (desjejum, almoço, lanche e jantar), as amostras das refeições servidas em cada instituição foram selecionadas para pesagem. Em cada instituição as bandejas foram marcadas e sorteadas três amostras (bandejas) em cada uma das refeições servidas durante o dia, abrangendo o consumo alimentar de todos os dias da semana. Para determinar o porcionamento médio para cada idoso calculou-se a média de peso de cada alimento/preparação oferecido durante a semana, dividindo-se pelo número de vezes em que foi servido. A tomada de peso foi feita em balança eletrônica digital, marca Marter, com capacidade de até 1000 g. Os líquidos foram medidos em recipientes de vidro com subdivisões de 50 $\mathrm{ml}$ e capacidade para até $250 \mathrm{ml}$. O cálculo do tamanho da amostra $(\mathrm{n}=21)$ representou $40,38 \%$ do universo selecionado $(n=156)$, correspondendo ao porcionamento médio para 63 idosos, determinado, basicamente, em função dos recursos disponíveis para a realização da pesquisa.

O modo de preparo e a distribuição dos alimentos foram observados pelos pesquisadores. Também procedeu-se a entrevistas com funcionários das instituições sobre alguns aspectos relacionados aos alimentos e prepara- 
ções servidos aos idosos. A aceitação ou a não aceitação do alimento/preparação pelos idosos foi avaliada a partir da observação de sobras no prato e da investigação do motivo da rejeição quando isso acontecia.

\subsection{Composição Nutricional das Dietas}

Para determinar a quantidade diária de energia e de nutrientes nas refeições servidas aos idosos, os valores médios obtidos para alimento/preparação foram analisados no programa de computação "Sistema de Apoio à Decisão em Nutrição", desenvolvido pela Universidade Federal de São Paulo. Para algumas preparações, como "batidinho", "cuscuz" e "Maria Isabel”, foram utilizadas as tradicionais Tabelas de Composição Química de Alimentos de Franco (1992) e IBGE (1996) para serem acrescidas ao programa de computador.

\subsection{Adequação da Dieta}

Para a avaliação da adequação da ingestão de energia, proteínas, vitamina $\mathrm{C}$ e ferro foram usadas como referencial as recomendações nutricionais preconizados pelo Food and Nutrition Board da National Academy of Sciences para as Recommended Dietary Allowances (1989). Com relação à adequação de cálcio e fósforo, foram usados os valores do National Academy of Sciences para Dietary Reference Intakes (DRIs, 1997).

\subsection{Análise Estatística}

Os dados foram representados por média, desvio-padrão e porcentagens de adequação, calculados utilizando programa Epi Info, versão 6.04.

\section{Resultados e Discussão}

A distribuição das pessoas idosas segundo sexo e faixa etária em cada instituição pesquisada está apresentada na Tabela 1. A idade do grupo de idosos abrigados nas instituições variou de 60 a 95 anos, 64,0\% apresentaram idade entre 70 e 90 anos e 18,0\% entre 60 e 70 ou entre 90 e 95 anos. Verificou-se que as mulheres representaram a maioria da população institucionalizada (70,5\%). Esses dados confirmam a sobrevida maior das mulheres em relação aos homens. 
TABELA 1 - Distribuição das pessoas idosas por faixa etária e sexo, em cada instituição pesquisada

\begin{tabular}{|c|c|c|c|c|c|c|c|}
\hline \multirow{2}{*}{$\begin{array}{l}\begin{array}{l}\text { Faixa } \\
\text { e tária } \\
\text { (anos) }\end{array} \\
60 \rightarrow 0\end{array}$} & \multicolumn{2}{|c|}{$\begin{array}{c}\text { In s titu iça o l } \\
\text { Mas culin o } \\
\text { Fem in in } 0\end{array}$} & \multicolumn{2}{|c|}{$\begin{array}{c}\text { In s titu ição } \\
\text { Masculino } \\
\text { Fem in in o }\end{array}$} & \multicolumn{2}{|c|}{$\begin{array}{c}\text { In s titu iça a III } \\
\text { Mas cu lin o } \\
\text { Fem in in o }\end{array}$} & \multirow{2}{*}{$\begin{array}{c}\text { TOTA L } \\
28 \\
(17,9 \%)\end{array}$} \\
\hline & $\begin{array}{c}4 \\
(8,7 \%)\end{array}$ & $\begin{array}{c}6 \\
(13 \%)\end{array}$ & $\begin{array}{c}5 \\
(6,3 \%)\end{array}$ & $\begin{array}{c}10 \\
(12,7 \%)\end{array}$ & $\begin{array}{c}1 \\
(3,2 \%)\end{array}$ & $\begin{array}{c}2 \\
(6,5 \%)\end{array}$ & \\
\hline $70-80$ & $\begin{array}{c}7 \\
(15,2- \\
\%)\end{array}$ & $\begin{array}{c}12 \\
(26,7- \\
\%)\end{array}$ & $\begin{array}{c}7 \\
(8,9 \%)\end{array}$ & $\begin{array}{c}20 \\
(25,3 \%)\end{array}$ & $\begin{array}{c}3 \\
(9,7 \%)\end{array}$ & $\begin{array}{c}9 \\
(29 \%)\end{array}$ & $\begin{array}{c}54 \\
(34,6 \%)\end{array}$ \\
\hline $80 \rightarrow 0$ & $\begin{array}{c}3 \\
(6,5 \%)\end{array}$ & $\begin{array}{c}10 \\
(21,7- \\
\%)\end{array}$ & $\begin{array}{c}4 \\
(5,1 \%) \\
\end{array}$ & $\begin{array}{c}16 \\
(20,2 \%)\end{array}$ & $\begin{array}{c}4 \\
(12,9 . \\
\%)\end{array}$ & $\begin{array}{c}6 \\
(19,3- \\
\%)\end{array}$ & $\begin{array}{c}46 \\
(29,5 \%)\end{array}$ \\
\hline $90 \rightarrow 95$ & $\begin{array}{c}2 \\
(4,4 \%)\end{array}$ & $\begin{array}{c}2 \\
(4,4 \%)\end{array}$ & $\begin{array}{c}4 \\
(5,1 \%)\end{array}$ & $\begin{array}{c}13 \\
(16,4 \%)\end{array}$ & $\begin{array}{c}2 \\
(6,5 \%)\end{array}$ & $\begin{array}{c}4 \\
(12,9 . \\
\%)\end{array}$ & $\begin{array}{c}28 \\
(17,9 \%)\end{array}$ \\
\hline TOTA L & $\begin{array}{c}16 \\
(34,8- \\
\%)\end{array}$ & $\begin{array}{c}23 \\
(65,2- \\
\%)\end{array}$ & $\begin{array}{c}20 \\
(25,4- \\
\%)\end{array}$ & $\begin{array}{c}59 \\
(74,6 \%)\end{array}$ & $\begin{array}{c}10 \\
(32,3- \\
\%)\end{array}$ & $\begin{array}{c}21 \\
(67,7- \\
\%)\end{array}$ & $\begin{array}{c}156 \\
(100 \%)\end{array}$ \\
\hline
\end{tabular}

Na investigação sobre os alimentos/preparações consumidos pelos idosos institucionalizados foi identificada em duas instituições a oferta de seis refeições diárias: desjejum, lanche, almoço, lanche, jantar e lanche noturno. Na terceira instituição eram oferecidas quatro refeições: desjejum, almoço, lanche e jantar.

O desjejum geralmente era composto de café com leite, açúcar ou adoçante para os diabéticos, pão com margarina, bolacha ou cuscuz. Estes componentes alimentares presentes no desjejum assemelham-se àqueles já verificados por Marucci (1985) em estudos de dietas para idosos institucionalizados. Os lanches sempre incluíam frutas ou suco de frutas acompanhado de bolachas, mingaus, café com leite e pão com margarina ou bolacha. No almoço, os alimentos/preparações mais usados foram arroz, feijão, "Maria Isabel", carne bovina, frango ou ovos, hortaliças e macarrão. O jantar era muito semelhante ao almoço, às vezes incluindo os mesmos alimentos usados no desjejum ou nos lanches. O lanche noturno era constituído de chá ou leite com bolacha.

No Quadro 1 encontra-se a relação dos alimentos e/ou preparações mais consumidos pelos idosos, de acordo com o número de vezes em que foram oferecidos nos dias da pesquisa. Observa-se que alimentos como arroz, café com leite, carne bovina, frango e feijão foram os únicos servidos nas três instituições. Outros alimentos como abóbora, mingau e pão doce 
apareceram em duas instituições, enquanto batata, biscoito, chá, cuscuz, farinha de mandioca, frutas, macarrão, "Maria Isabel", margarina, quiabo, chuchu, beterraba e risoto só foram servidos em uma instituição.

As diferenças encontradas na oferta dos alimentos entre as instituições estão mais relacionadas à disponibilidade de gêneros alimentícios por questões financeiras verificadas na época da realização do estudo. A variabilidade entre as porções servidas inter e intra-indivíduos provavelmente está mais ligada às inadequações dos porcionamentos dos alimentos no momento da distribuição das refeições servidas aos idosos. Observou-se que não havia uniformização dos utensílios empregados para a distribuição dos alimentos, faltou conhecimento dos funcionários para servir porções de tamanho correto visando atender às necessidades nutricionais do idoso e houve ausência de nutricionista nas instituições. Além disso, era comum e evidente o mau humor dos funcionários responsáveis pelo preparo e distribuição dos alimentos na maioria das instituições, havia um número reduzido de funcionários nas instituições e não existia programa de treinamento ou de qualificação de recursos humanos destinados aos cuidados no preparo, armazenamento, higienização e distribuição dos alimentos para as pessoas idosas. Analisando-se detalhadamente os alimentos preparados que integravam as refeições das pessoas idosas nas instituições, observou-se que as carnes mais consumidas foram a bovina e a de frango. A carne bovina foi oferecida na forma de picadinho, batidinho, almôndegas, sopas e "Maria Isabel". O frango geralmente foi servido ao molho, provavelmente para facilitar a mastigação e a digestibilidade, estimular o apetite e evitar a monotonia, já que o idoso apresenta problemas dentários, diminuição nas percepções de gosto, cheiro e visão, além de doenças e uso de drogas que possam reduzir o apetite e diminuir a absorção de nutrientes, influenciando negativamente no consumo alimentar e, conseqüientemente, nutricional. 
QUADRO 1 - Média e desvio padrão dos alimentos e/ou preparações mais servidos na semana segundo as intituições geriátricas

\begin{tabular}{|c|c|c|c|}
\hline Alimentos/Preparações & Instituição 1 & Instituição 2 & Instituição 3 \\
\hline Arroz cozido $(\mathrm{g})$ & $368,99 \pm 22,64$ & $268,16 \pm 32,86$ & $194,60 \pm 23,29$ \\
\hline Abóbora cozida (g) & $4,40 \pm 0,56$ & - & $14,80 \pm 1,35$ \\
\hline Batata doce cozida (g) & - & $164,17 \pm 0,0$ & - \\
\hline Batata inglesa cozida (g) & & & $7,28 \pm 1,50$ \\
\hline Beterraba (g) & $15,00 \pm 8,00$ & - & - \\
\hline Biscoito salgado (g) & - & $40,83 \pm 2,02$ & - \\
\hline Café com leite (ml) & $250,00 \pm 0,0$ & $244,00 \pm 1,00$ & $261,00 \pm 2,00$ \\
\hline Carne bovina/frango (g) & $119,00 \pm 10,00$ & $169,0 \pm 5,50$ & $93,00 \pm 3,50$ \\
\hline Cenoura (g) & - & - & $21,00 \pm 4,40$ \\
\hline Chás (ml) & - & $170,00 \pm 12,00$ & - \\
\hline Chuchu (g) & - & - & $20,00 \pm 10,00$ \\
\hline Cuscuz (g) & - & $130,00 \pm 4,40$ & - \\
\hline Farinha de mandioca $(\mathrm{g})$ & $25,50 \pm 6,40$ & - & - \\
\hline Feijão com verduras (g) & $73,00 \pm 2,50$ & $124,00 \pm 1,40$ & $46,00 \pm 6,00$ \\
\hline Frutas $(\mathrm{g})$ & - & - & $218,00 \pm 1,80$ \\
\hline Macarrão cozido (g) & - & $49,00 \pm 4,90$ & - \\
\hline Maria Isabel (g) & - & $334,0 \pm 4,20$ & - \\
\hline Mingaus (g) & - & $268,00 \pm 7,50$ & $250,00 \pm 1,20$ \\
\hline Pão doce (g) & - & $45,00 \pm 5,00$ & $63,00 \pm 2,6$ \\
\hline Quiabo (g) & $3,90 \pm 1,20$ & - & - \\
\hline Risoto(g) & - & $290,00 \pm 3,00$ & - \\
\hline Suco de fruta (ml) & - & $240,00 \pm 6,60$ & $158,00 \pm 6,20$ \\
\hline
\end{tabular}


Outra preparação muito empregada para amenizar os problemas relacionados à ingestão alimentar dos idosos foi o mingau, utilizando diferentes farináceos (Neston, Mucilon de arroz ou milho, Maizena, Cremogena, entre outros).

O arroz era servido simples ou misturado com carne, formando o prato típico conhecido como "Maria Isabel". Contudo, existem opções de enriquecê-lo com vegetais como cenoura, repolho, pimentão, tomate, cebola, coentro, ervilha, feijão e outras leguminosas, tornando-o mais nutritivo, saboroso e atrativo.

As verduras e os legumes foram utilizados em preparações de sopas, ficando mais tenros e facilitando a mastigação e a deglutição. Convém salientar que muitos idosos apresentam dificuldades de mastigação pela falta de dentes ou inadaptação ao uso de prótese, como já relatado por outros autores (NAJAS et al., 1991). As sopas sempre foram bem aceitas pelos idosos do presente estudo; além da de legumes, eram preparadas com carnes, feijão ou macarrão. Outros estudos mostram que sopa de verduras é freqüentemente consumida por muitos idosos em outras instituições. (CORREA; DUARTE, 1991).

Vale lembrar que se deve estar atento com relação aos cuidados que devem ser dispensados no preparo de sopas, porque o modo de elaboração e a cocção dos alimentos usados nesta preparação podem alterar o valor nutricional das verduras e hortaliças, que são fontes de vitaminas, minerais, fibras e antioxidantes.

Vitaminas, minerais e substâncias alimentares antioxidantes são essenciais ao bom desempenho de diversas reações químicas que ocorrem no organismo humano e devem fazer parte de uma dieta balanceada. Os efeitos benéficos destas substâncias já são bastante evidenciados na melhoria de muitas disfunções orgânicas inerentes ao processo de envelhecimento. É recomendado, portanto, garantir a sua presença no planejamento e na ingestão alimentar dos idosos.

A quantidade adequada de alimentos fontes de fibras tem importante função na dieta para minimizar problemas de doenças cardiovasculares, intestinais, diabetes e obesidade. Talvez devido aos problemas dentários ou ao desconhecimento da sua importância por parte dos responsáveis pela alimentação, tais alimentos foram pouco contemplados na dieta dos idosos estudados neste trabalho e, quando integrados aos cardápios, a preferência era 
pela oferta em preparações cozidas, moídas ou trituradas, nas quais o teor de fibras pode ser afetado.

As frutas predominantes foram laranja, acerola e abacaxi, mais usadas na forma de sucos, enquanto banana, mamão e melão foram mais usadas como vitaminas (leite + fruta + cereal industrializado). O suco de caju engarrafado foi muito utilizado por ser bem aceito pelos idosos. Ressalte-se que a preferência de utilização das frutas na forma de sucos e vitaminas pode ser decorrente de problemas dentários, bastante comuns nesta faixa etária. Em geral, os idosos apresentam ausência de dentes ou os têm em mau estado de conservação (CORREA; DUARTE, 1991).

Os vegetais mais utilizados foram chuchu, beterraba, cenoura, abóbora e batata, servidos como saladas cozidas ou preparações com carnes e sopas.

Também estiveram presentes preparações como "chás", podendo refletir o hábito alimentar do idoso ou, então, representar alguma forma de medicação. No estudo de Hiramatsu et al. (1991), os autores mostraram que 50,4\% dos medicamentos auto-administrados incluíam "chás" e analgésicos. No entanto, é bom estar atento, porque o uso de "chás" pode ser feito em substituição ao leite pela diferença de preço considerável entre os dois produtos (MARUCCI, 1992) ou pela intolerância ao leite em função da má digestão da lactose.

Muitos pesquisadores reconhecem que o cálcio é o mineral mais adequado na dieta de muitos idosos (MARUCCI, 1985; ALENCAR et al., 1991). Esse aspecto é relevante e deve ser considerado em todos os estudos sobre alimentação e nutrição de idosos, visto que o cálcio é nutriente importante para o desempenho dos sistemas cardiovascular e muscular, participa do processo de coagulação sanguínea, do transporte de oxigênio e de substâncias no nível de membrana plasmática. A deficiência de cálcio na dieta aumenta os riscos de frequiência de fraturas e de futura osteoporose, além de predispor ao câncer de cólon e à hipertensão arterial (LEE et al., 1996).

Com relação às gorduras, fizeram-se presentes na forma de óleo vegetal para o preparo das refeições ou de margarinas para consumo em pães ou cuscuz. Em geral, a preocupação predominante em relação ao papel dos lipídeos na dieta é quanto ao teor de colesterol e aos níveis totais de gordura para os idosos com doenças cardiovasculares. Já quanto ao interesse sobre a melhor proporção entre os diferentes ácidos graxos dentro do consumo total de gorduras, tanto para os idosos saudáveis quanto para os doentes, 
não houve maior atenção.

Muitas preparações estavam condicionadas à situação econômica, consistência, facilidade no preparo das refeições e hábito alimentar. A atenção quanto ao valor nutritivo e ao poder nutricional dos alimentos parece não haver sido percebida por todos os membros integrantes das instituições geriátricas. De um modo geral, as considerações sobre o consumo alimentar eram mais destacadas para limitar ou evitar o uso de determinado alimento pelos efeitos que a sua ingestão poderia causar sobre determinada doença. Por exemplo, açúcar para o diabetes, colesterol e gordura para o coração, sal para a hipertensão arterial. Não foram percebidos destaques para as características organolépticas dos alimentos, como cor e sabor, nem para a preocupação com o valor nutritivo deles.

Muitos idosos manifestam tendência à rejeição voluntária ao consumo de certos alimentos por tabus alimentares, doenças ou medicamentos. Apesar disto, não havia uma preocupação por parte das instituições em estimular e incentivar o consumo de alimentos de valor medicinal. Uma opção é a introdução de alimentos como a soja que, além de sua atividade antiestrogênica, possui diversas propriedades funcionais que podem proteger contra várias doenças crônicas.

Uma atenção nas formas de apresentação das refeições através da combinação de cor, textura, formas e aparências seria importante, tendo em vista que os alimentos poderiam ficar mais atraentes e contribuir para incentivar o consumo daqueles que trariam mais benefícios nutricionais à saúde. Assim, discutir as relações que existem entre as disponibilidades de alimentos nas instituições geriátricas, as preferências e as rejeições, aliando-se às possibilidades de inclusão de novos alimentos e preparações, amplia a possibilidade de se atingir a oferta de uma alimentação saudável para esta parcela da população. Na Tabela 2 encontra-se o consumo médio de energia e nutrientes nas instituições pesquisadas com os percentuais de adequação dispostos no gráfico. 
TABELA 2 - Média porcentual e desvio padrão do consumo médio, por dia, de energia e nutrientes, de acordo com as instituições geriátricas

\begin{tabular}{|c|c|c|c|}
\hline Nutrientes & Ins tituição l l (\%) & Instituição 2 (\%) & Ins tituição 3 (\%) \\
\hline Energia (kcal) & $1,563 \pm 2274 \%$ & $2,822 \pm 55134 \%$ & $2,196 \pm 45 \quad 104 \%$ \\
\hline Proteínas (\%) & 16 & 17 & 12 \\
\hline Lipideos (\%) & 23 & 25 & 35 \\
\hline Carboidrato $(\%)$ & 61 & 55 & 53 \\
\hline Cálcio (\%) & 95 & 139 & 140 \\
\hline Fósforo (\%) & 82 & 114 & 91 \\
\hline Ferro $(\%)$ & 150 & 190 & 140 \\
\hline Vitam ina $C(\%)$ & 70 & 77 & 52 \\
\hline
\end{tabular}

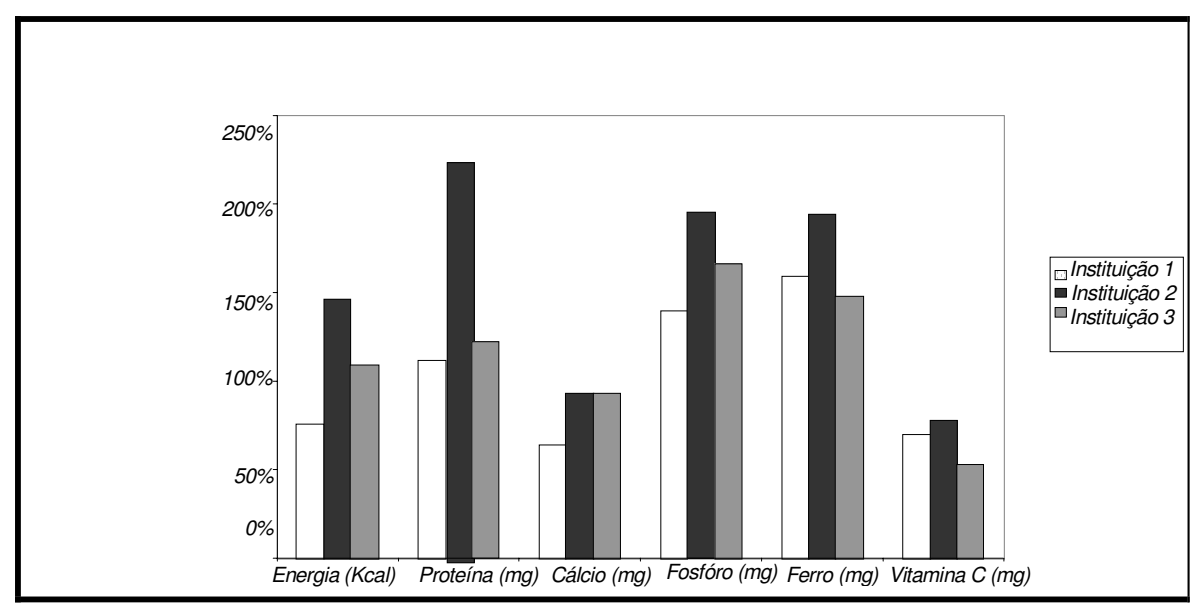

GRÁFICO 1: Adequação média de energia e nutrientes (\%) da dieta oferecida aos idosos em diferentes instituições geriátricas, segundo as recomendações americanas

Para os idosos pesquisados da Instituição 1 observa-se ingestão energética diária inferior ao recomendado. Contudo, o mesmo consumo mostrou-se superior nas outras duas instituições. As diferenças podem ser devidas a porcionamento, uso de preparações com densidades energéticas muito diferentes entre si, baixo grau de conscientização dos cuidadores quanto ao preparo das refeições e dos próprios idosos em relação às suas necessi- 
dades nutricionais, rejeição entre tipo/preparação ou consistência, uso de medicamentos ou doenças que podem afetar o consumo de alimentos.

Sabe-se que a necessidade energética modifica-se com a idade pelas mudanças apresentadas na composição corporal decorrente do envelhecimento e também pela redução de atividade física, devido principalmente a fatores culturais, levando a uma redução nas necessidades energéticas. A ingestão calórica excessiva pode levar ao ganho de peso e a alterações metabólicas adversas, as quais podem afetar a saúde, havendo maior prevalência de hipertensão arterial, diabetes, aterosclerose e problemas cardiocirculatórios.

A proteína ingerida pelos idosos apresentou valor superior ao recomendado, chegando a alcançar proporções bem mais elevadas do que a de energia (Gráfico 1). O excesso de proteínas pode comprometer o balanço de cálcio e ter um efeito negativo na saúde do osso (SELLMEYER et al., 2001).

O nutriente que se mostrou abaixo das recomendações em todas as instituições foi a vitamina $C$. Em contrapartida, o consumo médio de fósforo e de ferro excedeu as recomendações. A maior ingestão de fósforo em relação à de cálcio pode afetar seriamente o metabolismo do segundo nutriente (CALVO; PARK, 1996).

Ao verificar-se o consumo de cálcio, houve diferenças entre as três instituições, ficando clara a limitação na oferta deste mineral na Instituição 1; entre as Instituições 2 e 3, a oferta de cálcio não diferiu, tendo as duas apresentando valores próximos às recomendações. Uma relação da baixa ingestão de cálcio está associada à taxa aumentada de perda da massa óssea, tanto para animais quanto para humanos, diminuindo a densidade óssea (EGGER et al., 1994).

Um fato preocupante em relação à inadequação de cálcio nas dietas dos idosos é quando isso se associa a um consumo excessivo de fósforo ou de proteínas. A diminuição no consumo de cálcio e o elevado teor de fósforo propiciam uma relação cálcio/fósforo desfavorável, contribuindo para o aumento no risco de fraturas e de osteoporose. A proteína, quando em excesso, pode afetar negativamente o balanço de cálcio através de um aumento de sua excreção via urina (KERSTETTER; ALLEN, 1994), daí ser benéfica a oferta adequada de cálcio em equilíbrio com proteínas e fósforo para manter a densidade mineral óssea.

As elevadas concentrações de ferro observadas no conteúdo das refeições, aliadas ao baixo consumo de vitamina $\mathrm{C}$ e de antioxidantes são preocupantes, visto que o idoso é mais predisposto à formação de radicais 
livres e a deficiência prolongada destas substâncias aumenta a predisposição às infecções. Muitos são os benefícios que podem ser alcançados, incluindo a saúde do cérebro com o uso de alimentos fontes de nutrientes antioxidantes.

A vitamina $\mathrm{C}$ desempenha diversas funções em muitas reações e processos metabólicos, dentre eles a formação do colágeno, a síntese de epinefrina, corticosteróides e ácidos biliares. Além de co-fator enzimático, aumenta a absorção do ferro não-heme, age como antioxidante extracelular protegendo as células dos danos causados pelos radicais livres e inibindo a formação de nitrosaminas (MAYES, 1994).

A deficiência bioquímica de ácido ascórbico foi observada nos estudos de Van Der Wielen et al. (1994) em 65\% e 59\% de homens e mulheres idosos institucionalizados, respectivamente. O estudo populacional americano, Nhanes II - National Health and Nutrition Examination Survey, constatou, entre os indivíduos idosos, maior risco para inadequação nutricional em vitamina C. Também Asciutti-Moura (1987) observou diminuição dos teores circulantes de vitamina $\mathrm{C}$ em idosos institucionalizados, mesmo com ingestão satisfatória da vitamina.

O ácido ascórbico é amplamente encontrado em frutas cítricas e nos vegetais folhosos, muitos deles de baixo custo e com periodicidade constante durante o ano, devendo tornar-se presentes no cardápio do idoso.

É importante enfatizar que a deficiência de vitaminas em idosos institucionalizados não é apenas um problema de consumo alimentar. A forma de preparo do alimento, as doenças crônicas e o uso de medicação precisam ser também considerados (ARANHA et al., 2000).

\section{Considerações Finais}

Os resultados mostram diferenças marcantes na adequação alimentar e nutricional entre as dietas oferecidas nas instituições geriátricas de Teresina. A Instituição 2 diferiu muito da situação alimentar encontrada nas outras instituições, apresentando média superior de consumo de energia e demais nutrientes.

Dentro de cada instituição, os idosos recebem alimentação insuficiente ou com excesso de energia, acompanhada por consumo excedente de proteínas. Nutrientes importantes como cálcio e vitamina $\mathrm{C}$ apresentaram consumo insuficiente, sendo que o ferro e o fósforo atingiram valores acima das recomendações nacionais. 
Os resultados sugerem a necessidade de dispensar melhores cuidados nutricionais à dieta desses indivíduos, promovendo a contribuição para a melhoria de suas condições de nutrição e de saúde. Para tanto, recomendase que sejam feitos o treinamento e a capacitação dos funcionários visando estabelecer o seguimento de técnicas mais adequadas de preparo e distribuição de refeições, adoção de novas preparações e alimentos como formas de melhorar a qualidade nutricional das dietas, o compromisso dos familiares, do Município e do Estado como formas de garantir as condições adequadas de alimentação.

Por fim, é de grande importância pesquisar e analisar a composição da dieta dos idosos a fim de garantir o suprimento adequado em quantidade e qualidade de nutrientes, visto que as pessoas idosas constituem uma população de risco em função das peculiaridades de seus hábitos alimentares e de suas condições de saúde biológicas, físicas e psicológicas.

Novas investigações são necessárias para identificar o estado nutricional e as condições de saúde dos idosos.

\title{
EVALUATION OF NUTRICIONAL QUALITY OF THE MEALS SERVED FOR THE ELDERLY PEOPLE IN THE GERIATRICS INSTITUTIONS
}

\begin{abstract}
The food of elderly people living in three geriatric institutions in TeresinaPI, Brazil, was studied in order to evaluate the nutritional quality of the meals served. Direct weight method was used aiming to determine the average proportion of the food and its preparation. The caloric and nutritional values of the meals or preparations served were determined through the means of a computerized calculation program of diets developed by the Federal University of São Paulo. The adjustment of calories, proteins, iron and vitamin $\mathrm{C}$ was carried out based on the parameters of the National Council Research found in the Recommended Dietary Allowances (1989), while the adjustment of calcium and
\end{abstract}


phosphorus was done according to the Recommended Dietary Intake (1998). The results indicated inadequate caloric consumption in two of the institutions, being excessive in one and deficient in the other $(146.42 \%$ and $75.68 \%$ ), and protein intake above the recommendation in all of them $(112.23 \% ; 218,88 \%$ and $122.32 \%)$. The calcium intake was low $(63.57 \%)$ in one institution and adequate in the others, while the intake of vitamin $\mathrm{C}$ was low in all of them. The consumption of iron and phosphorus was high, presenting values above the recommendation. The inadequate diets offered to the elderly suggest the necessity of taking intervention measures in the short run, since unbalanced diets bring terrible consequences to the health, mainly to the elderly.

Keywords: Elderly People. Diet. Nutritional Adjustment. Institution.

\section{REFERÊNCIAS}

ALENCAR, Y. M. G.; CARVALHO FILHO, E. T.; RONCADA, M. J.; HIRAMATSU, C. S.; PIRES, E. A. C.; QUENTAL, I. A. Comportamento do Colesterol Total (CT) e suas Frações HDL e LDL em Idoso. In: CONGRESSO BRASILEIRO DE GERIATRIA E GERONTOLOGIA, 9., 1991, São Paulo. Resumos... São Paulo: Sociedade Brasileira de Geriatria e Gerontologia, 1991. (Tema livre, n. 115).

ARANHA, F. Q.; BARROS, Z. F.; MOURA, L. S. A.; GONÇALVES, M. C. R.; BARROS, J. C.; METRI, J. C.; SOUZA, M. S. O Papel da Vitamina C sobre as Alterações Orgânicas do Idoso. Revista de Nutrição, Campinas, v. 13, n. 2, p. 89-97, 2000.

ASCIUTT-MOURA, L. S. Evaluation du statut nutricional vitaminique $B_{1}, B_{2}, B_{6}, C$, A et e chez des personnes ágées en hospitalisation de longue durée. 1987. Thése (Doctorat en Gériatrie)- Université de Bourgogne, Dijon, 1987. $175 \mathrm{p}$.

BORN, T. Cuidado ao Idoso em Instituição. In: NETTO, M. P. Gerontologia, São Paulo: Atheneu, 1997. P. 403-413.

BRASIL. SEMINÁRIO INTERNACIONAL ENVELHECIMENTO POPULACIONAL: uma agenda para o final do século, 1., 1996, Brasília. Anais... Brasília: MPAS, 1996. 97 p.

CALVO, M. S.; PARK, Y. K. Changing Phosphorus Content of the U.S. 
Diet: potential for adverse on boné. Journal of Nutrition, Philadelphia, v. 126, p. 1168S-1180S, 1996.

CORRÊA, N. C.; DUARTE, A. L. N. Atividade da Nutricionista. In: JACOB FILHO, W. Relatório da Prática em Evolução do Grupo de Assistência Multidisciplinar ao Idoso Ambulatorial (GAMIA). São Paulo: HC/FMUSP/ BAYER, 1991. P. 20-21.

EGGER, C. D.; MUHLBAUER, R. C.; FELIX, R.; DELMAS, P. D.; MARKS, S. C.; FLEISCH, H. Evaluation of Urinary Pyridinium Crosslonk Excretion as a Marker of Boné Resorption in the Rat. Journal Bone and Mineral Research, New York, v. 9, n. 8, p. 1211-1219, 1994.

FRANCO, G. Tabela de Composição Química de Alimentos. 9. ed. São Paulo: Atheneu, 1992. 137 p.

GLOTH, F. M.; TOBIN, J. D.; SMITH, C. E.; MEYER, J. N. Nutrient Intakes in a Frail Homebound Elderly Population in the Community vs a Nursing Home Population. Journal American Dietetic Association, Chicago, v. 96, n. 6, p. 605-607, 1996.

GUIZOG, Y; VELLAS, B; GARRY, P. J. Assessing the Nutritional Status of the Elderly: the mini nutrition assessment as part of the geriatric evaluation. Nutrition Reviews, New York, v. 54, n. 1, p. 59-65, 1996.

HIRAMATSU, C. S.; ALENCAR, Y. M. G.; RONCADA, M. J.; CARVALHO FILHO, E. T.; MESSIAS, M. P.; MARUCI, M. F. N. Diagnósticos Clínicos em Pacientes de Idade Avançada. In: CONGRESSO BRASILEIRO DE GERIATRIA E GERONTOLOGIA, 9., 1991, São Paulo. Resumos... São Paulo: Sociedade Brasileira de Geriatria e Gerontologia, 1991. (Tema livre, n. 55).

IBGE. Tabelas de Composição de Alimentos. 4. ed. Rio de Janeiro, 1996. (Estudo nacional da despesa familiar).

Censo Demográfico 2000: características da população e domicílios. Rio de Janeiro, 2000. (Resultado do universo).

KERSTETTER, J. E.; ALLEN, L. H. Protein Intake and Calcium Homeostasis. Advances in Nutritional Reseach, New York, v. 9, n. 1, p. 167-181, 1994.

LEE, W. T.; LEUNG, S. S.; LEUNG, D. M.; CHENG, J. C. A Follow-up Study on the Effects of Calcium-supplement Withdrawal and Puberty on Bone Acquisition of Children. American Journal of Clinical Nutrition, New York, v. 64, p. 71-77, 1996. 
MARUCCI, M. F. M. Aspectos Nutricionais e Hábitos Alimentares de Idosos, Matriculados em Ambulatório Geriátrico. 1992. Tese. (Doutorado em Nutrição) - Faculdade de Saúde Pública, Universidade de São Paulo (USP), São Paulo,1992.

MARUCCI, M. F. M. Avaliação das Dietas Oferecidas em Instituições para Idosos, Localizadas no Município de São Paulo. 1985. Dissertação.(Mestrado em Nutrição) - Faculdade de Saúde Pública, Universidade de São Paulo (USP), São Paulo, 1985.

MAYES, P. A. Estrutura e Função das Vitaminas Hidrossolúveis. In: MURRAY, R. K.; GRANNER, D. K.; MAYS, P. A. Harper Bioquímica. 7. ed. São Paulo: Atheneu, 1994. P. 582-596.

NAJAS, M. S.; SACHS, A.; WAJSBERG, M.; SOUZA, A. L. M.; TONIOLO, J. N.; EBEL, S. J.; RAMOS, L. R., TUDISCO, E. S. Condição Bucal de Idosos e sua Relação com Habilidade de Mastigação e seu Estado Nutricional. In: CONGRESSO NACIONAL DE ALIMENTAÇÃO E NUTRIÇÃO, 2., 1991, São Paulo. Resumos... São Paulo: Sociedade Brasileira de Alimentação e Nutrição, 1991. (Tema livre, n. 86)

ORTEGA, R. M.; GARRIDO, G.; TURRERO, E.; CHAMORRO, M.; ALBO, E. D.; ANDRES, P. Valoración antropométrica del estado nutricional de un colectivo de ancianos de Madrid (España). Archivo Latinoamericano of Nutrition, Caracas, v. 42, p. 26-35, 1992.

SELLMEYER, D. E.; STONE, K. I.; SEBASTIAN, A.; CUMMINGS, S. R. For the Study Osteoporotic Fractures Research Griuo: a high ration of dietary animal to vegetable protein increases the rate of bone loss and the risk of fracture in postmenopausal women. American Journal of Clinical Nutrition, New York, v. 73, p. 118-122, 2001.

USA. NATIONAL ACADEMY OF SCIENCES. Dietary References Intakes. Nutrition Reviews, New York, v. 55, n. 9, p. 332-334, 1997.

USA. NATIONAL COUNCIL RESEARCH. Recommended Dietary Allowances. 10. ed. Washington, DC: National Academy Press, 1989. 